\title{
ÍNDICE PERCENTUAL DE RECONHECIMENTO DE SENTENÇAS NO SILÊNCIO E NO RUÍDO: EFEITOS DA ACLIMATIZAÇÃO NO INDIVÍDUO AVALIADO SEM AS PRÓTESES AUDITIVAS
}

\section{Percentual indexes of sentences recognition in quiet environment and under noise: effects of acclimatization in an individual evaluated without hearing aids}

\author{
Sinéia Neujahr dos Santos ${ }^{(1)}$, Tiago Petry ${ }^{(2)}$, Maristela Julio Costa ${ }^{(3)}$
}

\begin{abstract}
RESUMO
Objetivo: verificar o efeito da aclimatização no reconhecimento de sentenças no silêncio e no ruído, em novos usuários de próteses auditivas, avaliados sem as mesmas, antes e após o período de aclimatização. Métodos: participaram do estudo 40 indivíduos, idade entre 28 e 78 anos, que possuíam perda auditiva neurossensorial de grau leve a moderadamente severo, e estavam iniciando o processo de seleção e adaptação de próteses auditivas. Os testes foram realizados em diferentes sessões: antes da adaptação das próteses auditivas, 14 dias e três meses após a adaptação. Foi aplicado o teste Listas de Sentenças em Português (COSTA, 1997), em campo livre, no silêncio e no ruído, ambos em intensidade fixa de $65 \mathrm{~dB} A$, na qual foram obtidos os índices percentuais de reconhecimento de sentenças no silêncio (IPRSS) e no ruído (IPRSR). Resultados: os valores médios obtidos para o IPRSS na 1믈 $2^{a}$ e $3^{\text {a }}$ sessões foram, respectivamente, $65 \% ; 72 \%$ e $83 \%$ dB A. Já as médias dos IPRSR para a 1를 $2^{a}$ e $3^{\text {a }}$ sessões foram $51 \% ; 58 \%$ e $59 \%$ dB A. Ao comparar os resultados obtidos entre as sessões, foi verificada diferença estatisticamente significante, para o IPRSS, entre $1^{\underline{a}}$ e a $3^{\underline{a}}$ e entre a $2^{\underline{a}}$ e a $3^{\underline{a}}$ sessão e, para o IPRSR, entre a $1^{\underline{a}}$ e a $2^{\underline{a}}$ e entre a $1^{\underline{a}}$ e a $3^{\text {a }}$ sessão. Conclusão: os indivíduos melhoraram seu desempenho ao longo do uso das próteses auditivas, mesmo sendo avaliados sem as mesmas e essa melhora pode estar relacionada ao efeito da aclimatização.
\end{abstract}

DESCRITORES: Auxiliares de Audição; Aclimatação; Testes de Discriminação da Fala; Perda Auditiva; Plasticidade Neuronal

(1) Fonoaudióloga clínica da Sonora - Centro de Audiologia e Adaptação de Próteses Auditivas, Santa Maria, RS, Brasil; Mestre em Distúrbios da Comunicação Humana pela Universidade Federal de Santa Maria.

(2) Fonoaudiólogo; Professor Assistente do Curso de Fonoaudiologia do Centro Universitário Feevale, FEEVALE, Novo Hamburgo, RS, Brasil; Mestre em Distúrbios da Comunicação Humana pela Universidade Federal de Santa Maria.

(3) Fonoaudióloga; Professora Adjunta do Departamento de Fonoaudiologia da Universidade Federal de Santa Maria, UFSM, Santa Maria, RS, Brasil; Doutora em Ciências dos Distúrbios da Comunicação Humana pela Universidade Federal de São Paulo.

Conflito de interesses: inexistente

\section{INTRODUÇÃO}

A deficiência auditiva prejudica imensamente a qualidade de vida dos indivíduos e sua integração na sociedade. Em função dela, muitos alteram sua estrutura de vida, o que acarreta prejuízos afetivos, profissionais e no relacionamento com amigos e familiares ${ }^{1}$. Considerada uma doença severamente incapacitante, a deficiência auditiva não acomete apenas a audibilidade dos sons, mas também a qualidade da recepção, manifestando-se nos indivíduos como dificuldade de compreensão de fala, 
principalmente em ambientes ruidosos, comprometendo seriamente a qualidade de vida de seus portadores ${ }^{1,2}$.

Dessa forma, minimizar os efeitos limitadores causados por essa deficiência tornou-se possível, por meio da utilização de próteses auditivas. Elas têm por finalidade restabelecer a audibilidade dos sons de fala, que são percebidos com menos intensidade e muitas vezes com menor qualidade, dependendo do tipo da lesão, melhorando assim a sua comunicação ${ }^{3}$.

Quando é indicada uma prótese auditiva a um indivíduo com perda, espera-se que além de melhorar a sua capacidade de perceber os sons, também ocorra uma melhora na compreensão de fala, uma vez que a prótese auditiva reintroduz a estimulação auditiva de sons que o indivíduo não era mais capaz de ouvir naturalmente ${ }^{4}$. Essa estimulação auditiva periférica vai permitir, portanto, que as vias auditivas centrais se reorganizem e passem a produzir efeitos positivos nas habilidades auditivas, refletindo em uma melhora na compreensão dos sons da fala, fenômeno chamado de aclimatização ${ }^{5,6}$. Essa reorganização das vias chama-se plasticidade auditiva e refere-se à capacidade de ocorrerem mudanças anatômicas e/ou funcionais no sistema responsável pela transmissão das informações auditivas.

Muitos estudos têm como objetivo verificar se a aclimatização ocorre, mas a maioria deles avalia seus indivíduos sempre com a utilização de próteses auditivas. Contudo, se esse fenômeno realmente acontece, ele poderia ser quantificado no indivíduo mesmo sem a utilização dos aparelhos auditivos. $\mathrm{O}$ indivíduo, frente a uma mesma situação de comunicação, melhoraria seu desempenho, quando avaliado sem a utilização das próteses auditivas?

Diante deste questionamento, o presente estudo tem como objetivo verificar a ocorrência da aclimatização, por meio dos limiares de reconhecimento de sentenças no silêncio e no ruído, em indivíduos com perda auditiva neurossensorial, desde o início do processo de adaptação de próteses auditivas, durante e após o período de aclimatização, sem a utilização das próteses auditivas.

\section{MÉTODOS}

O presente estudo trata-se de uma pesquisa observacional, longitudinal, descritiva e quali-quantitativa. Foi realizado no Laboratório de Próteses Auditivas do Serviço de Atendimento Fonoaudiológico (SAF) da Universidade Federal de Santa Maria. Esta pesquisa é um subprojeto dentro de um projeto intitulado: Pesquisa em Base de Dados em Saúde Auditiva.
Participaram do estudo somente indivíduos que concordaram com a realização dos procedimentos necessários para a execução da pesquisa e assinaram o Termo de Consentimento Livre e Esclarecido, após terem recebido maiores esclarecimentos sobre o objetivo e metodologia do estudo proposto.

Para fazerem parte da pesquisa, os critérios de seleção estabelecidos foram: ter idade igual ou superior a 18 anos, diagnóstico audiológico de perda auditiva do tipo neurossensorial de grau leve a moderadamente severo, com Limiar de Reconhecimento de Fala (LRF) de no máximo 65 $\mathrm{dB}$ na melhor orelha, nunca ter utilizado próteses auditivas, fazer parte do Programa de Doação de Próteses Auditivas do Governo Federal e estar em processo de adaptação binaural dos aparelhos auditivos. Como critério de exclusão, considerou-se qualquer indivíduo que, por algum motivo, não aceitou participar da pesquisa, não cumpriu os critérios de seleção ou apresentou algum fator que poderia interferir no teste, como alterações neurológicas e/ ou de fluência verbal.

Os sujeitos que participaram do estudo foram avaliados no período que compreendeu o mês de janeiro de 2008 a janeiro de 2009. Todos os indivíduos que estavam no início do processo de seleção e adaptação de próteses auditivas e preencheram os critérios de inclusão acima descritos, foram previamente selecionados. Dentre, aproximadamente, 200 pacientes atendidos, 47 foram selecionados $\mathrm{e}$ destes, 40 concluíram todas as avaliações.

No total, fizeram parte do estudo 40 sujeitos, com idade média de 62 anos, com mínimo de 28 e máximo de 78 anos. Destes, 23 mulheres e 17 homens.

Os indivíduos foram submetidos à pesquisa dos Índices Percentuais de Reconhecimento de Sentenças no Silêncio e no Ruído (IPRSS e IPRSR), em campo livre.

Os testes foram realizados sem a utilização das próteses auditivas, em diferentes sessões de avaliação, consistindo em três sessões para cada sujeito. A primeira avaliação foi realizada antes da adaptação das próteses auditivas, a segunda quatorze dias após a adaptação e a terceira três meses após a adaptação dos aparelhos auditivos.

O IPRSS e o IPRSR foram obtidos utilizandose o teste Listas de Sentenças para o Português ${ }^{7}$ (LSP), constituído por uma lista de 25 sentenças, outras sete listas com 10 sentenças e um ruído com espectro de fala. As sentenças e o ruído estão gravados em $C D$, em canais independentes.

As medidas desta pesquisa foram obtidas em cabine tratada acusticamente, utilizando um audiômetro digital de dois canais, marca Damplex, modelo DA65; e um sistema de amplificação para audiometria em campo livre, modelo TA 1010. As 
sentenças foram apresentadas utilizando-se um CD Player na marca Britânia, modelo B5279, na opção lineout acoplada ao audiômetro.

A aplicação do teste foi realizada em ambiente acusticamente tratado, em campo livre e com o indivíduo posicionado a um metro da fonte sonora, de frente para a mesma, a 0 - -0 - azimute.

Inicialmente, foi realizado um treinamento para que os sujeitos se familiarizassem com o teste. As dez primeiras sentenças da lista $1 \mathrm{~A}$ foram aplicadas aos indivíduos antes da obtenção dos IPRSS. $\mathrm{Na}$ obtenção dos IPRSR foi utilizado o mesmo procedimento, porém na presença de ruído competitivo.

$A$ intensidade das sentenças permaneceu sempre $65 \mathrm{~dB} A$, intensidade escolhida em função de ser frequentemente utilizada em situações habituais de conversação, já que a fala possui uma intensidade média de 60 a 65 dB NPS, com picos de máxima ou mínima intensidade variando cerca de $30 \mathrm{~dB}^{1}$.

$\mathrm{Na}$ pesquisa do IPRSR, o ruído permaneceu constante a $65 \mathrm{~dB} \mathrm{~A}{ }^{7}$, determinando uma relação S/R fixa de zero.

Apesar de o equipamento ter sido previamente calibrado conforme as normas técnicas, os estímulos de fala e de ruído foram monitorados durante toda a pesquisa. Para isto, foi usado um medidor digital do nível de pressão sonora (NPS), marca Radio Schak, para determinar e garantir sempre as mesmas condições acústicas para todos os sujeitos avaliados. Foi selecionada a escala $A$ do medidor do NPS, com respostas rápidas, que é a mais utilizada na mensuração de ruídos contínuos e para determinar valores extremos de ruídos intermitentes, além de ser a escala usada pela autora do teste LSP, utilizado nesta pesquisa.

O procedimento utilizado para calibração do canal das sentenças foi realizado através do tom puro presente na primeira faixa do CD (canal 1). O uso do tom puro é necessário, pois a fala é um som complexo, que apresenta uma variação de 30 $\mathrm{dB}$ entre o som mais intenso e o menos intenso, oscilando $12 \mathrm{~dB}$ acima e $18 \mathrm{~dB}$ abaixo da média ${ }^{8}$. Portanto, o uso de um som contínuo de referência mantém sempre as mesmas condições de apresentação. Já a calibração do ruído foi realizada por meio do mesmo, presente no canal 2 .

A saída de cada canal foi calibrada através do VU-meter do audiômetro. Tanto o tom puro, presente no canal 1 , quanto o ruído, presente no canal dois, foram ajustados no nível zero.

Esta pesquisa teve aprovação do Comitê de Ética do gabinete de projetos do Centro de Ciências da Saúde sob no 0138.0.243.000-06.

Após o cálculo dos IPRSS e IPRSR, estes foram analisados e comparados estatisticamente. As variáveis foram comparadas duas a duas, nas diferentes sessões de avaliação: 1a sessão - antes da adaptação dos aparelhos auditivos, $2^{2}$ sessão - 14 dias após adaptação dos aparelhos auditivos e $3^{\text {a }}$ sessão - três meses após adaptação dos apareIhos auditivos. Após constatar a normalidade dos dados, foi aplicado o teste para a diferença entre duas amostras dependentes, o teste t pareado, que verifica se as diferenças encontradas entre as sessões de avaliação tiveram significância ou não. Foi considerado resultado significante $p \leq 0,05$, marcados com asterisco $\left(^{*}\right)$. Também foi realizada uma análise descritiva dos dados, expondo os resultados entre as sessões de avaliação.

\section{RESULTADOS}

Nas Tabelas 1 e 3 estão apresentados a média, desvio padrão, valor máximo, mínimo e os resultados do teste t pareado entre as sessões de avaliação, respectivamente, para os IPRSS e IPRSR.

Nas Tabelas 2 e 4 estão o percentual de indivíduos que melhoraram e os valores de melhora dos IPRSS e IPRSR, entre as avaliações.

Tabela 1 - Média, desvio padrão, valores mínimos, máximos e resultado do teste t pareado, para os IPRSS, entre as sessões de avaliação, para os indivíduos que não obtiveram $100 \%$ de acertos na $1^{1}$ avaliação $(\mathrm{N}=13)$

\begin{tabular}{ccccccc}
\hline Variáveis & Sessão & Média & DP & Mín & Máx & Valor de p \\
\hline \multirow{2}{*}{ IPRSS } & $1^{\underline{a}}$ & $65 \%$ & 21,84 & 30 & 90 & 0,1161 \\
& $2^{\underline{a}}$ & $72 \%$ & 28,62 & 20 & 100 & \\
IPRSS & $2^{\underline{a}}$ & $72 \%$ & 28,62 & 20 & 100 & $0,0500^{*}$ \\
& $3^{\text {a }}$ & $83 \%$ & 21,75 & 30 & 100 & \\
\multirow{2}{*}{ IPRSS } & $1^{\underline{a}}$ & $65 \%$ & 21,84 & 30 & 90 & $0,0006^{*}$ \\
& $3^{\text {a }}$ & $83 \%$ & 21,75 & 30 & 100 & 0 \\
\hline
\end{tabular}

*Apresentaram diferença estatisticamente significante para o teste t pareado 
Tabela 2 - Percentual de sujeitos que melhoraram e os valores de melhora para os IPRSS, entre as sessões de avaliação, para os indivíduos que não obtiveram $100 \%$ de acertos na $1^{a \underline{a}}$ avaliação ( $\left.N=13\right)$

\begin{tabular}{|c|c|c|c|}
\hline Variáveis & $\mathbf{N}$ & Melhorou & $\begin{array}{l}\text { Valor médio de } \\
\text { melhora }\end{array}$ \\
\hline $1^{\circ}$ IPRSS X $2^{\circ}$ IPRSS & 7 & $53,8 \%$ & $21,4 \%$ \\
\hline $2^{\circ}$ IPRSS $\times 3^{\circ}$ IPRSS & 7 & $53,8 \%$ & $25,7 \%$ \\
\hline $1^{\circ}$ IPRSS $\times 3^{\circ}$ IPRSS & 12 & $92,3 \%$ & $20,8 \%$ \\
\hline
\end{tabular}

Tabela 3 - Média, desvio padrão, valores mínimos, máximos e resultado do teste t pareado, para o IPRSR, entre as sessões de avaliação, para todos os indivíduos avaliados ( $\mathrm{N}=40)$

\begin{tabular}{ccccccc}
\hline Variáveis & Sessão & Média & DP & Mín & Máx & Valor de p \\
\hline \multirow{2}{*}{ IPRSR } & $1^{\text {a }}$ & $51 \%$ & 24,28 & 0 & 100 & \multirow{2}{*}{$0,0022^{*}$} \\
& $2^{\underline{a}}$ & $58 \%$ & 25,57 & 0 & 100 & \\
IPRSR & $2^{\underline{a}}$ & $58 \%$ & 25,57 & 0 & 100 & 0,3622 \\
& $3^{\text {a }}$ & $59 \%$ & 25,03 & 0 & 100 & \\
IPRSR & $1^{\text {a }}$ & $51 \%$ & 24,28 & 0 & 100 & $0,0032^{*}$ \\
& $3^{\underline{a}}$ & $59 \%$ & 25,03 & 0 & 100 & \\
\hline
\end{tabular}

${ }^{*}$ Apresentaram diferença estatisticamente significante para o teste t pareado

Tabela 4 - Percentual de sujeitos que melhoraram e os valores de melhora para os IPRSR, entre as sessões de avaliação, para todos os indivíduos avaliados $(\mathrm{N}=40)$

\begin{tabular}{cccc}
\hline Variáveis & N & Melhorou & $\begin{array}{c}\text { Valor médio de } \\
\text { melhora }\end{array}$ \\
\hline 10 IPRSR $\times$ 2 IPRSR & 30 & $75 \%$ & $17 \%$ \\
20 IPRSR X 3ㅇ IPRSR & 23 & $57,2 \%$ & $17 \%$ \\
1 IPRSR X 3ㅇ IPRSR & 18 & $45 \%$ & $25 \%$ \\
\hline
\end{tabular}

\section{DISCUSSÃO}

A fim de facilitar a leitura e compreensão, a discussão será apresentada em duas partes, referentes aos IPRSS e IPRSR.

Índice percentual de reconhecimento de sentenças no silêncio (IPRSS)

Inicialmente, deve-se esclarecer que a estratégia empregada para a obtenção do IPRSS não foi sensível a todos os indivíduos, pois a intensidade de $65 \mathrm{~dB}$ A, utilizada na apresentação das sentenças, intensidade de fala frequentemente utilizada no dia-a-dia, foi favorável em função do grau da perda apresentado por 27 (67,5\%) dos 40 indivíduos avaliados. Estes sujeitos possuíam média tritonal inferior a intensidade utilizada na apresentação do estímulo, o que possibilitou o acerto de todas as sentenças apresentadas.
Esse fato permite afirmar que se deve basear no limiar de reconhecimento de sentenças no silêncio para a obtenção do índice percentual de reconhecimento de sentenças no silêncio, pois assim torna-se possível verificar qual a dificuldade apresentada pelo paciente, ou seja, em qual intensidade de fala ele não tem um bom desempenho e então, nesta mesma condição, avaliá-lo novamente após a intervenção.

Assim sendo, como 27 indivíduos apresentaram desempenho de $100 \%$ desde a primeira avaliação, não possibilitando mencionar a melhora da performance ao longo das duas avaliações subsequentes, apenas 13 sujeitos $(32,5 \%)$ apresentaram desempenho inferior a $100 \%$ na primeira sessão de avaliação, cujos resultados serão discutidos a seguir.

Os valores médios encontrados na $1^{\underline{a}}, 2^{\underline{a}}$ e $3^{a}$ sessão, nesses indivíduos, foram respectivamente $65 \% ; 72 \%$ e $83 \%$ (Tabela 1). 
Ao analisar esses valores, observa-se que, a cada avaliação realizada, a porcentagem de acertos foi maior do que a anterior, o que nos mostra melhora no desempenho dos indivíduos, mesmo quando avaliados sem a utilização dos aparelhos.

Ao comparar os resultados obtidos entre as sessões, foi verificada diferença estatisticamente significante entre a $2^{\underline{a}}$ e a $3^{\underline{a}}$ e entre a $1^{\underline{a}}$ e $3^{\underline{a}}$ a sessão de avaliação (Tabela 2).

Pode-se atribuir os resultados constatados entre a $2^{\underline{a}}$ e a $3^{\underline{a}}$ e entre a $1^{\underline{a}}$ e a $3^{\underline{a}}$ sessão de avaliação à aclimatização perceptual auditiva, que ocorre em um tempo médio de aproximadamente três meses após a adaptação das próteses auditivas ${ }^{2,9}$.

A utilização das próteses possibilitou maior estimulação auditiva a partir da amplificação, implicando em uma possível melhora na recepção e organização dos estímulos sonoros ${ }^{4}$, ocasionada pela reorganização das vias auditivas. Isso se reflete em um aperfeiçoamento no reconhecimento da fala ao longo do tempo ${ }^{5}$. A aclimatização seria progressiva e decorrente da melhor utilização de pistas acústicas, fornecidas pelo uso das próteses auditivas ${ }^{10}$.

Quando analisado o percentual de sujeitos que melhoraram seu desempenho entre as sessões de avaliação, foi observado que $53,8 \%$ obtiveram melhora entre a $1^{\text {a }}$ e a $2^{\text {a }}$ sessão; $53,8 \%$ entre a $2^{\text {a }}$ e a 3ㅜㅜ ; e 92,3\% entre a $1^{\underline{a}}$ e a $3^{\underline{a}}$ (Tabela 2).

Com base nesses achados, observa-se que a grande maioria melhorou entre a $1^{\text {a }}$ e a $3^{\text {a }}$ sessão, concordando com o período de tempo em que ocorre a aclimatização, segundo autores já anteriormente citados.

Ao analisar os sujeitos que melhoraram seu desempenho entre as avaliações, encontram-se valores médios de melhora, de $21,4 \%$ entre a 1 a e a $2^{\text {a }}$ sessão; $25,7 \%$ entre a $2^{\text {a }}$ e a $3^{\text {a }}$; e $20,8 \%$ entre a $1^{\text {a }}$ e a $3^{\text {a }}$ (Tabela 2).

Esses valores encontrados se assemelham aos encontrados na literatura. A aclimatização pode representar uma melhora entre zero e $10 \%$ no reconhecimento de fala ${ }^{2}$; em outro estudo, o aperfeiçoamento no reconhecimento da fala, ao longo do tempo de uso dos aparelhos auditivos pode refletir em uma melhora de $20 \%$ no desempenho do indivíduo ${ }^{9}$.

Pode-se observar que a maioria dos indivíduos melhorou seu desempenho entre a $1^{\underline{a}}$ e a $3^{\underline{a}}$ sessão de avaliação, com média de melhora de $20,8 \%$. Estes sujeitos foram de $65 \%$ para $83 \%$ de acertos, o que denota uma compreensão de, aproximadamente, $100 \%$ dos estímulos apresentados, já que cada frase repetida corretamente representa $10 \%$.

\section{Índice percentual de reconhecimento de} sentenças no ruído (IPRSR)

Os valores médios obtidos para a $1^{\underline{a}}, 2^{\underline{a}}$ e $3^{\underline{a}}$ sessão foram $51 \%$; $58 \%$ e $59 \%$ (Tabela 3 ).

Ao observar estes resultados, nota-se uma melhora entre as sessões, nos mostrando que os indivíduos tiveram um desempenho mais satisfatório após a experiência com as próteses auditivas.

Com base nestes achados, pode-se supor que ao estimular o sistema auditivo através da reintrodução do estímulo sonoro, as pistas acústicas relacionadas aos sons da fala ficariam um pouco mais evidentes. Isso proporcionaria um melhor desempenho frente a uma situação de conversação diária, em que tanto fala quanto ruído estariam a uma intensidade de $65 \mathrm{~dB} A$, situação na qual o indivíduo, antes da protetização, apresentou maior dificuldade de compreender a mensagem, após a reintrodução da estimulação acústica, passou a apresentar melhor resultado, mesmo quando não estava usando os aparelhos.

Ao comparar os resultados obtidos entre as sessões, foi verificada que a diferença estatisticamente significante ocorreu entre a $1^{\underline{a}}$ e a $2^{\underline{a}}$ e entre a $1^{\underline{a}} \mathrm{e}$ a $3^{\text {a }}$ sessão (Tabela 3 ), indicando que um melhor desempenho no reconhecimento de fala, proporcionado pelo uso das próteses auditivas, pode acontecer a partir da segunda semana de uso. De acordo com alguns autores ${ }^{11}$, as melhorias representadas pela aclimatização no reconhecimento de fala não ocorrem imediatamente nos novos usuários de próteses auditivas. Segundo alguns estudos, a aclimatização pode ocorrer após aproximadamente três meses após a adaptação das próteses auditivas ${ }^{9}$. Já outros pesquisadores referem que pode ocorrer seis meses após a adaptação ${ }^{10}$, variar entre um mês e meio e três meses após o uso da amplificação ${ }^{2}$, ou até ocorrer a partir do primeiro mês de adaptação ${ }^{12}$.

Ao analisar o percentual de sujeitos que melhoraram seu desempenho entre as sessões de avaliação, foi observada melhora de $75 \%$ dos sujeitos entre a $1^{\text {a }}$ e a $2^{\text {a }}$ sessão; $57,2 \%$ entre a $2^{\underline{a}}$ e a $3^{\text {a }}$; e $45 \%$ entre a $1^{\text {a }}$ e a $3^{\text {a }}$ (Tabela 4$)$.

Com base no número de sujeitos que aumentaram sua porcentagem de acertos, entre as sessões de avaliação, observamos que a maior parte deles melhorou entre a $1^{\underline{a}}$ e a $2^{\underline{a}}$ avaliação, o que poderia não ser consequência da aclimatização, já que este efeito ocorre em um período mínimo de um mês de utilização dos aparelhos auditivos ${ }^{13}$. Isto sugere que outros fatores podem estar agregados a estes melhores resultados, como o próprio aprendizado espontâneo do indivíduo a utilizar as novas informações acústicas ou seu entusiasmo frente os 
possíveis benefícios proporcionados pelas próteses auditivas ${ }^{10,13}$.

Ao analisar os sujeitos que melhoraram seu desempenho entre as avaliações, foram encontrados valores médios de melhora, para o IPRSR, de $17 \%$ entre a $1^{\mathrm{a}}$ e a $2^{\mathrm{a}}$ sessão, $17 \%$ entre a $2^{\mathrm{a}}$ e a $3^{\mathrm{a}}$; e $25 \%$ entre a $1^{a}$ e a $3^{\text {a }}$ (Tabela 4 ).

Por sua vez, poder-se-ia atribuir os melhores resultados entre a $1^{\underline{a}}$ e a $3^{\underline{a}}$ sessão de avaliação à aclimatização perceptual auditiva. A utilização das próteses reintroduziu a estimulação auditiva a partir da amplificação, aprimorando as habilidades que contribuem para a compreensão de fala. Isso se deve a plasticidade neural, que se refere à capacidade das vias auditivas centrais de se reorganizarem em resposta à estimulação auditiva. Esta reorganização leva a ocorrência de uma melhora no reconhecimento da fala dos usuários de próteses auditivas em longo prazo. À medida que o indivíduo aprende a utilizar as novas pistas de fala disponíveis com o uso da amplificação, ele compreende a fala mais facilmente ${ }^{6}$. A aclimatização seria progressiva e decorrente da melhor utilização de pistas acústicas, fornecidas pelo uso das próteses auditivas ${ }^{12,14}$.

A aclimatização implica na melhora da habilidade para utilizar as informações acústicas, em conseqüência da experiência auditiva. $O$ melhor desempenho está associado à adaptação das próteses auditivas ${ }^{12,14}$. Na tentativa de explicar essas modificações perceptuais que ocorrem nos usuários de próteses auditivas, pesquisadores ${ }^{15}$ hipotetizaram que a reabilitação auditiva de adultos com perdas auditivas neurossensoriais envolve a plasticidade funcional, na qual o sistema nervoso central pode se reorganizaria de acordo com as mudanças ambientais. Usuários de prótese auditiva, quando comparados a não usuários, apresentavam melhor desempenho em tarefas de discriminação da fala e sensação de intensidade, atribuindo essa melhora à plasticidade neural auditiva.

A aclimatização pode representar uma melhora entre zero e $10 \%$ no reconhecimento de fala ${ }^{2}$. Em outro estudo, o autor mostrou provas que as vias auditivas podem ser modificadas mediante a utilização de próteses auditivas, estas reintroduzem o sinal acústico, estimulando as vias e melhorando o desempenho do indivíduo cerca de $20 \%{ }^{9}$.

O maior valor de melhora encontrado, para o IPRSR, foi de $25 \%$ entre a $1^{\underline{a}}$ e a $3^{\underline{a}}$ sessão de avaliação. Este valor, na situação de ruído, é de grande relevância para os indivíduos, pois mesmo sendo avaliados sem as próteses auditivas três meses após a adaptação, eles melhoraram sua porcentagem de acertos.

Na situação de ruído, a avaliação mostrou-se sensível a meta proposta, pois além da maioria dos indivíduos ter aumentado a porcentagem de acertos, em uma situação de ruído competitivo, essas porcentagens $(17 \%$ e $25 \%$ de melhora entre as sessões) são relevantes em ambientes ruidosos, onde a maioria das queixas é relatada, e com certeza, diminuem o incômodo de pedir para que a mensagem seja repetida pelo interlocutor, possibilitando assim, que o indivíduo possa compreender parte restante da mensagem pelo contexto.

\section{CONCLUSÃO}

De acordo com os dados encontrados, pode-se concluir que o uso das próteses auditivas, durante um período de quatorze dias a três meses, foi capaz de provocar uma melhora na capacidade de reconhecer a fala no silêncio e no ruído, nos os indivíduos avaliados sem a utilização das mesmas. 


\section{ABSTRACT}

Purpose: to check the effect of acclimatization on sentence recognition in quiet environment and under noise, in new users of hearing aids, evaluated without their use, before and after the acclimatization period. Methods: the study comprised 40 individuals, aged between 28 and 78year old with mild to moderate sensorineural hearing loss. They were starting the selection and adaptation process of hearing aids. The tests were conducted in three sessions, before the adaptation of hearing aids, fourteen days and three months later, respectively. they were applied to Portuguese Sentences Lists test (COSTA, 1997), in free field, in quiet environment and under noise, both in fixed $65 \mathrm{~dB}$ A intensity, in which we obtained the percentual indexes of sentences recognition in quiet environment (PISRQ) and under noise (PISRN). Results: the mean values for PISRQ in the 1st, 2nd and 3rd sessions were, respectively, $65 \% ; 72 \%$ e $83 \% \mathrm{~dB}$ A. The mean values of PISRN for the 1 st, 2 nd and 3rd sessions were $51 \% ; 58 \%$ e $59 \% \mathrm{~dB} A$, respectively. While comparing the results between sessions, there was a statistically significant difference for PISRQ between the 1st and 3rd e and between the 2nd and 3rd session, and for PISRN, between the 1st and 2nd e and between the 1st and 3rd session. Conclusion: the subjects improved their performance using hearing aids, even being evaluated without them, and this improvement may be related to the acclimatization effect.

KEYWORDS: Hearing Aids; Acclimatization; Speech Discrimination Tests; Hearing Loss; Neuronal Plasticity

\section{REFERÊNCIAS}

1. Almeida K. Avaliação dos resultados da intervenção. In: Almeida K, lório MCM. Próteses auditivas: fundamentos teóricos e aplicações clínicas. 2. ed. São Paulo: Lovise; 2003. p. 335-56.

2. Humes LE, Wilson DL, Barlow NN, Garner C. Changes in hearing-aid benefit following 1 or 2 years of hearing-aid use by older adults. J Speech Lang Hear Res. 2002; 45(4):772-82.

3. Bucuvic EC, lório MCM. Benefício e dificuldades auditivas: um estudo em novos usuários de prótese auditiva após dois e seis meses de uso. Fono Atual. 2004; 7(29):19-29.

4. Amorim RMC, Almeida K. Estudo do benefício e da aclimatização em novos usuários de próteses auditivas. Pró-Fono. 2007; 19(1):39-48.

5. Willott JF. Physiological plasticity in the auditory system and its possible relevance to hearing aid use, deprivation effects, and acclimatization. Ear Hear. 1996; 17(3Suppl):66S-77S.

6. Arlinger S, Gatehouse S, Bentler RA, Byrne D, Cox RM, Dirks DD, etal. Report of the Eriksholm Workshop on auditory deprivation and acclimatization. Ear Hear. 1996; 17(3Suppl):87S-98S.
7. Costa MJ. Lista de sentenças em português: apresentação e estratégias de aplicação na audiologia. Santa Maria: Pallotti; 1998.

8. Boothroyd A. Speech perception, sensorioneural hearing loss and hearing aids. In: Studebaker G, Hockberg I. Acoustical factors affecting hearing aid performance. 2. ed. Boston: Allyn \& Bacon; 1993. p. 277-99.

9. Munro KJ. Reorganization of the adult auditory system: perceptual and physiological evidence from monaural fitting of hearing aids. Trends Amplif. 2008; 12(3):245-71. http://dx.doi. org/10.1177/1084713808323483

10. Reber MB, Kompis M. Acclimatization in firsttime hearing aid users using three different fitting protocols. Auris Nasus Larynx. 2005; 32(4):345-51. http://dx.doi.org/10.1016/j.anl.2005.05.008

11. Yund EW, Roup CM, Simon HJ, Bowman GA. Acclimatization in wide dynamic range multichannel compression and linear amplification hearing aids. J Rehabil Res Dev. 2006; 43(4):517-36.

12. Prates LPCS, lório MCM. Aclimatização: estudo do reconhecimento de fala em usuários de próteses auditivas. Pró-Fono. 2006; 18(3):259-66.

13. Myers S, Palmer CV. Functional and subjective changes over 16 weeks after hearing aid fitting: a case study. Semin Hear. 2005; 26(3):140-3. 
14. Caporali AS, Silva JA. Reconhecimento de fala no ruído em jovens e idosos com perda auditiva. Rev Bras Otorrinolaringol. 2004; 70(4):525-32.
15. Philibert B, Collet L, Vesson JF, Veuillet E. Intensity-related performances are modified by long-term hearing aid use: a functional plasticity? Hear Res. 2002; 165(1-2):142-51.

DOI: 10.1590/S1516-18462010005000019

RECEBIDO EM: 16/08/2009

ACEITO EM: 13/12/2009

Endereço para correspondência:

Sinéia Neujahr dos Santos

Av. 2 de Novembro, 1414 ap. 303

Santa Maria - RS

CEP: 97020-230

E-mail: sineians@gmail.com

Rev. CEFAC. 2010 Set-Out; 12(5):733-740 